\title{
Studies of carbohydrate-protein interaction by capillary electrophoresis
}

\author{
A. Taga, S. Yasueda, M. Mochizuki, H. Itoh and S. Honda \\ Faculty of Pharmaceutical Sciences, Kinki University, Kowakae, Higashi-osaka 577, Japan
}

Capillary electrophoresis is a useful tool for estimation of the binding constant between small amounts of a protein and a carbohydrate.

\footnotetext{
$\mathrm{T}$
} he high capabilities of capillary electrophoresis in both separation and detection have been well recognized. It allows high resolution separation with theoretical plate numbers often exceeding several hundred thousands and reproducible quantification by on-capillary detection. In addition to these advantages, capillary electrophoresis has a characteristic feature that it can easily be performed in free solution, i.e., it is usually carried out in a single phase usually of aqueous solution. Therefore, one can observe physical as well as chemical reactions occurring in a capillary prior to or during electrophoretic analysis. Thus, sample components can be converted to desired derivatives in a capillary, which can be separated from accompanying substances by an appropriate mode and detected by a suitable method. We have classified this kind of derivatization (in-capillary derivatization) into three types, and discussed the advantages and disadvantages of individual types [1-3].

The present paper is a brief review including our recent papers of physical reaction between substances, taking protein/carbohydrate couples as model systems. The in-capillary technique is important, because it permits accurate estimation of association/dissociation constants using minute amounts of substances in media similar to physiological fluids. 


\section{Experimental}

Capillary electrophoresis was carried out mainly using an Applied Biosystems 270A apparatus, equipped with a vacuum injector and a UV detector. A roll of capillary $(50 \mu \mathrm{m}$ i.d., $375 \mu \mathrm{m}$ o.d.) was obtained from Polymicro Technologies (Phoenix, AZ, USA). Desired portions of this capillary were cut out from the roll and a window was made for each cut-out piece at the $50-\mathrm{cm}$ position from one end. Each of them was installed onto the apparatus by fixing the window at the center of the light path in the detector. The capillary was flushed with $1 \mathrm{M}$ sodium hydroxide for ample period of time before each series of measurements.

Sample solutions were introduced into a capillary by suction using the vacuum injector for specified periods of time from the anodic end, and analyzed at $30 \pm 0.1^{\circ} \mathrm{C}$. Detection was performed by monitoring UV absorption at $220 \mathrm{~nm}$.

\section{Results and discussion}

\section{Interaction of proteins with acidic carbohydrates}

When a protein sample is introduced to an electrophoretic solution containing an acidic carbohydrate and an electric field is applied, they interact with each other as illustrated in figure 1 .

The velocity of electrophoretic migration (VEPM) of the protein sample in the presence of the carbohydrate additive $\left(v_{\mathrm{p}}\right)$ is increased by coupling to this negatively charged carbohydrate. As electroosmotic flow is concurrently flowing to the opposite direction, the value of $v_{\mathrm{p}}$ is between the VEPM of the free protein sample $\left(V_{\mathrm{p}}\right)$ and the VEPM of the protein - acidic carbohydrate complex $\left(V_{\mathrm{p} \text {-ac }}\right)$, and can be expressed as follows [4].

$$
v_{\mathrm{p}}=\alpha \cdot V_{\mathrm{p}-\mathrm{ac}}+(1-\alpha) V_{\mathrm{p}}
$$

where $\alpha$ is the molar fraction of the complex. Since there is an equilibrium between a free protein and the complex,

$$
\alpha=K_{\mathrm{p}-\mathrm{ac}} \bullet C_{\mathrm{ac}}\left(K_{\mathrm{p}-\mathrm{ac}} \bullet C_{\mathrm{ac}}+1\right)^{-1}
$$

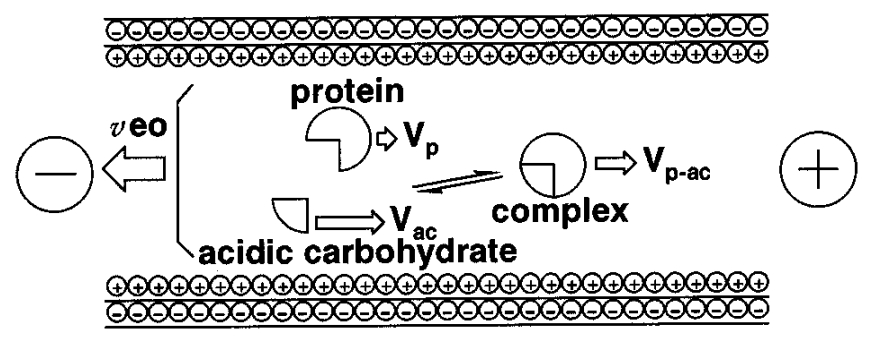

Figure 1. Illustration of the electrophoretic migration of a protein sample, an acidic carbohydrate as additive, and the complex formed from them in the direct method for association constant estimation. $v_{\mathrm{eo}}$ : velocity of electroosmotic flow, $V_{\mathrm{p}}$ : velocity of electrophoretic migration (VEPM) of the protein, $V_{\mathrm{ac}}$ : VEPM of the acidic carbohydrate, $V_{\text {p-ac }}$ : VEPM of the protein - acidic carbohydrate complex. where $K_{\mathrm{p}-\mathrm{ac}}$ is the association constant and $C_{\mathrm{ac}}$ is the concentration of the acidic carbohydrate.

Combination of equations (1) and (2), followed by replacement of velocity terms by migration times gives an important equation, as follows.

$\left(t-t_{1}\right)^{-1}=t_{2} \cdot t_{1}^{-1}\left(t_{2}-t_{1}\right)^{-1} K_{\mathrm{p}-\mathrm{ac}}^{-1} C_{\mathrm{ac}}{ }^{-1}+\left(t_{2}-t_{1}\right)^{-1}$.

In equation (3) $t$ and $t_{1}$ are the migration times of a protein sample in the presence and absence of an acidic carbohydrate as additive, respectively, and $t_{2}$ is the migration time of the complex, which can be estimated as the maximum migration time for sufficiently high concentrations of the additive. Equation (3) implies that the plot of the reciprocal of $\left(t-t_{1}\right) v s$ the reciprocal of $C_{\text {ac }}$ gives a straight line having a slope of $t_{2} \cdot t_{1}^{-1}\left(t_{2}-t_{1}\right)^{-1} K_{\mathrm{p}-\mathrm{ac}}^{-1}$ and the ordinate intercept of $\left(t_{2}-t_{1}\right)^{-1}$. Therefore, $K_{\mathrm{p} \text {-ac }}$ can be obtained as follows using the slope $(a)$ and the intercept $(b)$ of this line.

$$
K_{\mathrm{p}-\mathrm{ac}}=a^{-1} \cdot t_{1}^{-1}\left(b \cdot t_{1}+1\right) .
$$

Figure 2a shows an example of $t v s C_{\text {ac }}$ plot for a system composed of Ricinus communis hemagglutinin $\left(\mathrm{RCA}_{60}\right)$ (sample) - lactobionic acid (additive) system.

Figure $2 \mathrm{~b}$ demonstrates the linearity of the $\left(t-t_{1}\right)^{-1} \mathrm{vs}$ $C_{\mathrm{ac}}{ }^{-1}$ plot of this system. From the slope and the intercept of this line $K_{\text {p-ac }}$ was calculated as $3.3 \times 10^{3} \mathrm{M}^{-1}$. This value is a bit smaller than a reported value of the association constant of $\mathrm{RCA}_{60}$ to lactose $\left(1.5 \times 10^{4} \mathrm{M}^{-1}\right)$ [5]. It is reasonable because the reducing monosaccharide residue is oxidized to gluconic acid which less contributes to the interaction with this lectin.

The proposed method is accurate and reproducible. Repeated measurements of the association constant of
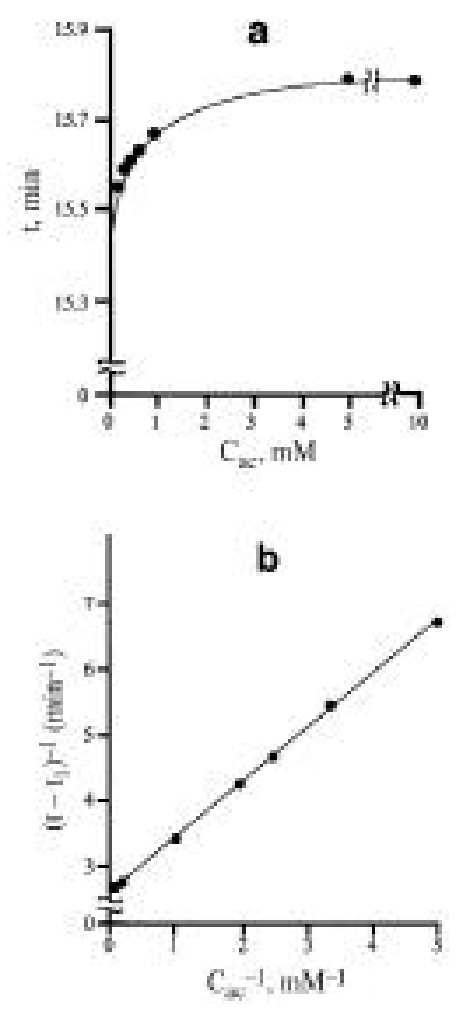

Figure 2. Plot of $t$ vs. $C_{\mathrm{ac}}$ a) and $t^{-1}$ vs. $C_{\mathrm{ac}}{ }^{-1}$ b) for the $\mathrm{RCA}_{60}-$ lactobionic acid system. 
$\mathrm{RCA}_{60}$ to lactobionic acid gave a relative standard deviation value of $3.6 \%$ for $n=5$. It requires only small amounts of sample. The minimum required amount of $\mathrm{RCA}_{60}$ in this system was as small as $10 \mathrm{pg}$ per injection. The amount of the acidic carbohydrate is larger, when the normal procedure is employed in which both a capillary and the electrode reservoirs are filled with the same buffer containing the same concentration of the acidic carbohydrate. Replacement of the electrode solutions in the reservoirs by mere buffer not containing the acidic carbohydrate reduces the amount of the acidic carbohydrate to a few thousandths. Thus, c.a. $5 \mu \mathrm{g}$ of lactobionic acid was enough in this series of measurement. In stronger interacting systems the required amount of additive will be further reduced depending on the magnitude of affinity.

This method has an additional advantage that it is independent of sample concentration. This was experimentally verified.

\section{Interaction of carbohydrates with neutral carbohydrates}

The foregoing argument is directed to a system involving an acidic carbohydrate as additive. In most cases, however, carbohydrates are electrically neutral, i.e., have no electric charge. In order to apply capillary electrophoretic measurement to such compounds, some devices are required. We could conquer this problem by two ways; one by chemical conversion to acidic carbohydrates and the other by a competitive binding technique.

\section{Introduction of ionic groups to neutral carbohydrates}

There are a number of methods for pre-capillary derivatization of carbohydrates, such as 2-aminopyridine [6], ethyl 4aminobenzoate [7], isoindole [8], and 1-phenyl-3-methyl-5pyrazolone methods [9]. Most of them are based on reductive amination. Only the 1-phenyl-3-methyl-5-pyrazolone method is based on another type of reaction (modified Knoevenagel condensation). All these methods introduce either acidic or basic group(s) to a neutral carbohydrate, but these groups absorb UV/VIS light or fluoresce strongly. For the studies of interaction, however, this absorbing and fluorescing properties are not welcome, because the derivatives give high base line levels even at low concentrations and low signal to noise ratios. We have developed a method which proceeds rapidly under mild conditions and the derivatives neither absorb nor fluoresce in the UV/VIS regions (Fig. 3).

This is based on dithioacetalation with 2-mercaptoethanesulfonate (MES) [10]. Two MES groups are introduced smoothly to the reducing end of each carbohydrate,

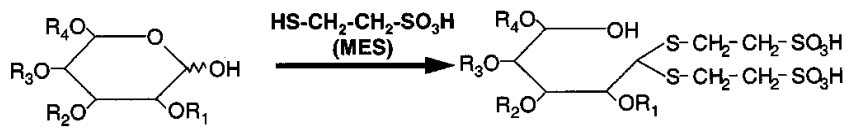

Figure 3. Introduction of the sulfonate groups to a reducing carbohydrate by dithioacetalation with MES. simply by adding the sodium salt of MES to an acidic solution of a carbohydrate, and the derivative has strong negative charge due to the introduction of the sulfonate groups. After simple cleanup of the derivative by evaporation and gel filtration, the product can be used as an additive to an electrophoretic solution as obtained. The yield is quantitative by use of anhydrous trifluoroacetic acid as reaction medium and by control of temperature at c.a. $25{ }^{\circ} \mathrm{C}$. Possible degradation in the cleanup process can be minimized by performing this process at low temperature around $0{ }^{\circ} \mathrm{C}$.

Estimation of the association constant of $\mathrm{RCA}_{60}$ to MESlactose gave a value of $2.47 \times 10^{3} \mathrm{M}^{-1}$ which is close to the association constant to lactobionic acid, demonstrating the reliability of this method of derivatization.

\section{Competitive binding with acidic carbohydrates}

Another method to make it possible to estimate the association constant of a protein to a neutral carbohydrate is competition of a neutral carbohydrate with an acidic carbohydrate having a similar structure, as illustrated in figure 4 [10].

If we consider a system in which an acidic carbohydrate is added to a buffer solution to a fixed concentration and a neutral carbohydrate is further added to various concentrations, we can obtain equation (5) based on the same principle as that used for derivation of equation (3).

$\left(t_{1}{ }^{\prime}-t^{\prime}\right)^{-1}=$

$t_{2}^{\prime}\left(t_{1}^{\prime}-t_{2}^{\prime}\right)^{-1} t_{1}{ }^{-1}\left(1+K_{\mathrm{p}-\mathrm{ac}} \cdot C_{\mathrm{ac}}\right) K_{\mathrm{p}-\mathrm{nc}}{ }^{-1} C_{\mathrm{nc}}{ }^{-1}+\left(t_{1}{ }^{\prime}-t_{2}^{\prime}\right)^{-1}(5)$

where $t^{\prime}$ is the migration time of the protein sample in the presence of the acidic carbohydrate at a fixed value of $C_{\mathrm{ac}}$ and the neutral carbohydrate at varying $C_{\mathrm{nc}}$ values. The term $t_{1}{ }^{\prime}$ designates the migration time of the protein for a special case where the acidic carbohydrate is at the fixed $C_{\mathrm{ac}}$ value but the neutral carbohydrate is not added $\left(C_{\mathrm{nc}}=0\right)$. The term $t_{2}{ }^{\prime}$ is the migration time for another special case where the acidic carbohydrate is not added. $K_{\text {p-ac }}$ is the association constant of the protein to the acidic carbohydrate. Its value is supposed to be already known from preliminary experiments using the direct method mentioned above. $K_{\mathrm{p}-\mathrm{nc}}$ is the association constant of the protein to the neutral carbohydrate, which we are going to estimate using this ternary system.

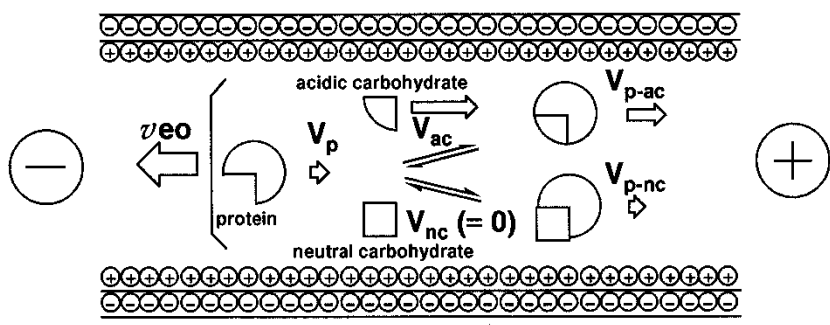

Figure 4. Illustration of the electrophoretic migration of a protein sample, an acidic and a neutral carbohydrates as additives, and the complexes formed from them in the competitive binding method for association constant estimation. $V_{\text {nc }}$ : VEPM of an neutral carbohydrate, $V_{\mathrm{p} \text {-nc }}$ : VEPM of the protein - neutral carbohydrate complex. The other symbols as in figure 1 . 
Equation (5) is rather complex as compared to equation (3), but there is also linearity for the $\left(t_{1}{ }^{\prime}-t^{\prime}\right)^{-1} v s C_{\mathrm{nc}}{ }^{-1}$ plot, and $K_{\mathrm{p}-\mathrm{nc}}$ can be obtained by equation (6) using the slope $\left(a^{\prime}\right)$ and the ordinate intercept $\left(b^{\prime}\right)$.

$$
K_{\mathrm{p}-\mathrm{nc}}=a^{\prime-1} \bullet t_{1}^{\prime-1}\left(b^{\prime} \bullet t_{1}^{\prime}-1\right)\left(K_{\mathrm{p}-\mathrm{ac}} \cdot C_{\mathrm{ac}}+1\right) .
$$

The obtained value of $K_{\mathrm{p}-\mathrm{nc}}$ for the $\mathrm{RCA}_{60}-$ lactose MES lactose system was $1.50 \times 10^{4} \mathrm{M}^{-1}$, which is in good agreement with the reported value obtained by the equilibrium dialysis method $\left(1.5 \times 10^{4} \mathrm{M}^{-1}\right)$.

In order to confirm the reliability of the competitive binding method, the association constant of antithrombin III to heparin was measured by the direct method and the competitive binding method using a binary system (antithrombin III - heparin) and a ternary system (antithrombin III heparin - low molecular weight heparin), respectively, since heparin is a convenient object of both methods and low molecular weight heparin used as a competing ligand is considered to interact with antithrombin III in the same fashion as heparin. The experimental value of the association constant of antithrombin III to heparin obtained by using equation (3) was $1.10 \times 10^{7} \mathrm{M}^{-1}$. In the competitive binding method a low molecular weight heparin sample was added to the electrophoretic solution to a concentration of $1.0 \times 10^{-6} \mathrm{M}$ and heparin was further added to the resultant solution to varying concentrations. The value of association constant of antithrombin III to heparin obtained by using equation (5) was $1.3 \times 10^{7} \mathrm{M}^{-1}$. These values obtained by both methods are in good agreement, demonstrating the reliability of the competitive binding method.

\section{Conclusions}

The association constant of a protein to an acidic carbohydrate could be conveniently estimated from the migration time difference of the protein added to an electrophoretic solution containing the acidic carbohydrate from that not containing the carbohydrate. This method is not dependent on sample concentration. It allows reproducible estimation and requires only small amounts of the protein and the carbohydrate. When the carbohydrate has no electric charge, it should be converted to the MES derivatives, which are suitable objects of the estimation of the association constant by the above-mentioned direct method. Addition of an acidic carbohydrate, for example the MES derivative of the neutral carbohydrate to be examined, to the protein - neutral carbohydrate system is also a useful technique to estimate the binding constant to a neutral carbohydrate. The reliability of this competitive binding method was confirmed by comparing the obtained value for antithrombin III to heparin in competition with low molecular weight heparin, with the value obtained by the direct method.

The systematic methods summarized in this paper are confined to the cases where a protein is a sample and a carbohydrate is an additive in an electrophoretic solution. This situation can be reversed, though a problem arises that reproducible migration time cannot be obtained, if an uncoated fused silica capillary is used. Coating the inner wall of the capillary with a hydrophilic polymer is essential. We describe the details of the reversed systems elsewhere.

\section{Acknowledgments}

A part of this work was supported by a grant for Carbohydrate Research Project of Kinki University in 1995.

\section{References}

1. Taga, A.; Honda, S. J. Chromatogr. 1996, 742, 243.

2. Taga, A.; Sugimura, M.; Honda, S. J. Chromatogr. 1998, 802, 243.

3. Taga, A.; Nishino; A., Honda; S. unpublished results (submitted to J. Chromatogr.).

4. Honda, S.; Taga, A.; Suzuki, K.; Suzuki, S.; Kakehi, K. J. Chromatogr. 1992, 597, 377.

5. Olsnes, S., Saltvedt, E., Bachhawat, B. K., Eur. J. Biochem. 1974, 44, 151.

7. Wang, W. T.; LeDonne, N. C., Jr.; Ackerman, B.; Sweeley, C. C. Anal. Biochem. 1984, 141, 366.

8. Lieu, J.; Shirota, O.; Wiesler, D.; Novotny, M. Proc. Nat. Acad. Sci., USA 1991, 88, 2302.

9. Honda, S.; Akao, E.; Suzuki, S.; Okuda, M.; Kakehi, K.; Nakamura, J. Anal. Biochem. 1989, 180, 351.

10. Honda, S.; Taga, A.; Yasueda, S. Presented at HPCE'95 (Würzburg, 1995). 